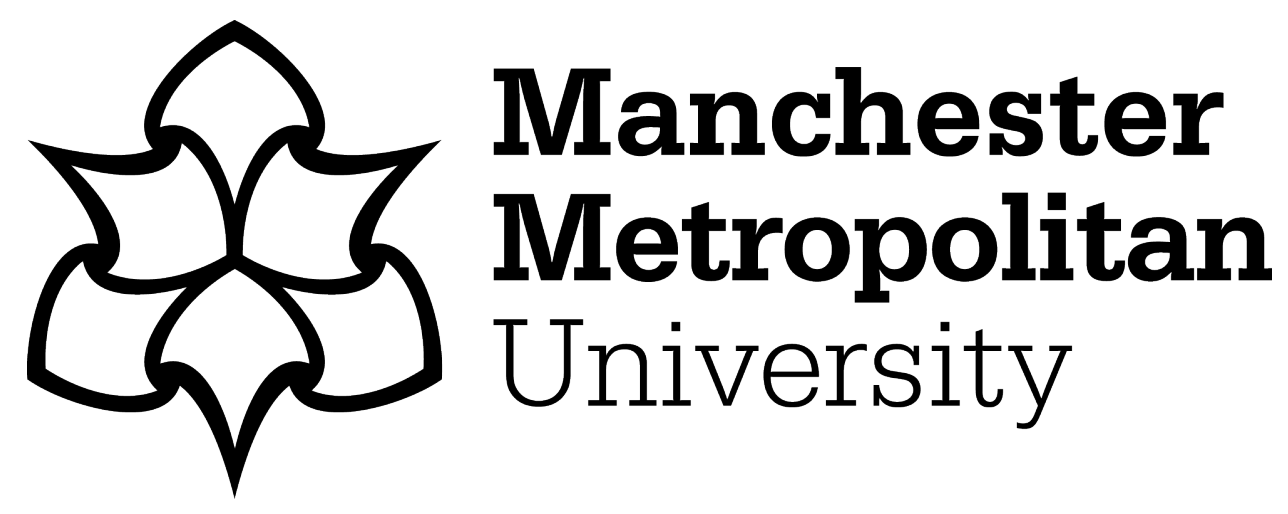

Zhang, D, Liu, Y, Dai, L, Bashir, AK, Nallanathan, A and Shim, B (2019) Performance Analysis of Decentralized V2X System with FD-NOMA. In: IEEE 90th Vehicular Technology Conference (VTC2019-Fall), 22 September 2019 - 25 September 2019, Honolulu, HI, USA.

Downloaded from: https://e-space.mmu.ac.uk/628392/

Version: Accepted Version

Publisher: IEEE

DOI: https://doi.org/10.1109/VTCFall.2019.8891360

Please cite the published version 


\title{
Performance Analysis of Decentralized V2X System with FD-NOMA
}

\author{
Di Zhang* ${ }^{* \dagger}$, Yuanwei Liu ${ }^{\ddagger}$, Linglong Dai ${ }^{\S}$ Ali Kashif Bashir ${ }^{\llbracket}$, Arumugam Nallanathan ${ }^{\ddagger}$, and Byonghyo Shim ${ }^{\dagger}$ \\ * School of Information Engineering, Zhengzhou University, Zhengzhou, China \\ † Information System Laboratory, EECS, Seoul National University, Seoul, Korea \\ $\ddagger$ EECS, Queen Mary University of London, London, U.K. \\ $\S$ Department of Electronic Engineering, Tsinghua University, Beijing, China \\ I School of Computing, Mathematics, and Digital Technology, Manchester Metropolitan University, Manchester, U.K.
}

\begin{abstract}
We introduce a full duplex non-orthogonal multiple access (FD-NOMA)-based decentralized vehicle to everything (V2X) system model and focus on its capacity performance analysis. In order to solve the computation complicated problems of the involved exponential integral functions and infinite factorial expressions, we give approximate closed-form expressions with controllable arbitrary small errors. We find the accuracy of our approximate expressions is controlled by the division of $\frac{\pi}{2}$ in the urban and crowded (UC) scenario, and the truncation point $T$ in the suburban and remote (SR) scenario. Numerical results manifest 1) Increasing the number of V2X device, NOMA power and Rician factor value yields better capacity performance. 2) Effect of FD-NOMA is determined by the FD self-interference and the channel noise. 3) FD-NOMA has better latency performance compared to other schemes.

Index Terms-Vehicle communications, V2X, full duplex, non-
\end{abstract} orthogonal multiple access, performance analysis.

\section{INTRODUCTION}

In terms of vehicle to everything (V2X) communications, the dedicated short-range communications (DSRC) [1] has been popular for decades. Recently, cellular V2X (C-V2X) [2] attracts growing attention with its ability to connect more devices and provide even faster transmission rate to these connected devices. However, the current version of C-V2X (i.e., the orthogonal frequency division multiple access-basced long term evolution V2X (OFDMA-based LTE-V2X)) cannot fully satisfy the requirements of low latency, various quality of services (QoSs) and different transmit rates [3], [4].

It is noticed that some fifth generation $(5 \mathrm{G})$ technologies can be used to satisfy the low latency, various QoS and different transmit rates requirements [5], [6]. For instance, comparing to the orthogonal multiple access (OMA) scheme, non-orthogonal multiple access (NOMA) can accommodate more users, and these users can be with different QoS requirements [7]. Another merit of NOMA is that it is insensitive to carrier frequency offset (CFO) effect caused by moving vehicles, which is especially useful for V2X communications. On the other hand, full duplex (FD) can provide faster speed and better spectrum efficiency (SE) performances compared to the half-duplex (HD) [7]. FD can also offer reliable communications [8], which is useful for V2X applications, e.g., the emergency message broadcasting. On combining the FD and NOMA schemes (i.e., FD-NOMA), authors in [9], [10] found that it can significantly suppress the co-channel interferences and achieve better performance gains compared to half duplex NOMA (HD-NOMA) and orthogonal multiple access (OMA).

In literature, some exisitng studies on NOMA-V2X and FDV2X [11]-[13] have been done. For instnace, the authors in [12] proposed the graph-based practical encoding and join$\mathrm{t}$ belief propagation (BP) decoding techniques, which can achieve any rate pair close to the capacity region. B. Di et al. in [11] employed NOMA for ultra-reliable low latency (URLLC) communications while proposing a NOMA-based mixed centralized/distributed (NOMA-MCD) scheme to reduce the resource collision. In [13], an optimal blind interference alignment scheme was proposed for the FD and HD coexisting modes. However, cellular base station (BS) plays the dominant roles in these studies, which is a challenge for connecting massive fast transmission rate $\mathrm{V} 2 \mathrm{X}$ devices due to the $\mathrm{BS}$ throughput restriction.

On the other hand, for the capacity analysis, various channel models are used, for instance, $\kappa-\mu$ channel model [14], [15], $\eta-\mu$ channel model [14]. Although the Rayleigh and Rician channel models have been widely adopted, it is intractable to obtain some closed-form expressions because of the involved exponential integral functions and infinite factorial expressions. Some approximate methods and algorithms have been introduced to solve these problems, for instance, the Swamee and Ohija method and the fast and accurate algorithm. However, these methods are based on some special conditions or with low accuracy.

Based on the aforementioned discussions, in this work, we try to answer the following key questions:

- Can we use FD-NOMA to meet the requirements of $V 2 X$ communications?

- If the FD-NOMA is satisfactory, what about its capacity performance?

- Is there some approximate expression for the ergodic capacity analysis of Rayleigh and Rician channel models with arbitrary small error and low computational complexity?

The main contributions of this study are given as follows: 
- The FD-NOMA-based decentralized V2X systems are proposed, which can partly offload the cellular network. Compared to prior regimes, FD-NOMA is insensitive to Doppler effect caused by moving vehicles, and can synchronously accommodate more users with different QoSs and transmit rates.

- We derive the exact system ergodic capacity expressions and their approximate closed-form expressions. These approximate closed-form expressions are with low computational complexity and controllable arbitrary small errors compared to the existing approximate expressions. We find 1) the accuracy of our simplified approximate expression in urban and crowded (UC) scenario is controlled by the associated division of $\frac{\pi}{2}$ (with respect to the exponential integral function $E_{1}(x)$ ). 2) The accuracy of our simplified approximate expression in suburban and remote (SR) scenario is controlled by the truncation point $T$ (with respect to the exponential integral function $E_{n}(x)$ ).

- Numerical results indicate: 1) The validity of our derivations (with Monte-Carlo simulations). 2) System capacity increases with the increasing allocated power value, signal to noise ratio (SNR) and Rician factor values. 3) FD selfinterference and the channel noise determine the effect of FD-NOMA.

\section{FD-NOMA-BASED DECENTRALIZED V2X SYSTEMS AND THE CAPACITY ANALYSIS}

\section{A. System Model}

The FD-NOMA-based decentralized V2X system model is given in Fig. 1. In this system, no relay is used because of the vehicle's limited energy. V2X devices can directly communicate with each other, and the required contents are obtained from neighboring V2X caches [17]. This system model thus are with shorter transmission distance and better latency performance [17], it can also reduce the cellular network load. In addition, all V2X devices (vehicle, pedestrian, traffic lights, etc.) are comprehensively included ${ }^{1}$. We further classify the V2X communications into two scenarios: 1) the UC scenario and 2) the SR scenario. In UC scenario, abundant reflection and refraction links between source and destination are existing [18]. In contrast, in SR scenario, there always exists one light of sight (LoS) link between the source and destination [19]. Additionally, compared to UC scenario, there are less obstacles in SR scenario.

We assume that transmission channels are uncorrelated in this study. The considered multiple input multiple output NOMA (MIMO-NOMA) links thus can be treated as a sum of additive single input single output NOMA (SISO-NOMA) links. Similar to prior literatures [7], [20], we adopt an increasing order of the channel gain, which means $\left|h_{i, 1}\right|^{2} \leq, \ldots,\left|h_{i, j}\right|^{2} \leq, \ldots, \leq$ $\left|h_{i, N}\right|^{2}, \forall i \in[1, M], j \in[1, N]$, vice versa. In this case, after successive interference cancellation (SIC), NOMA co-channel

\footnotetext{
${ }^{1}$ Here in Fig. 1, to simplify the figure, only vehicle to vehicle (V2V) and vehicle to infrastructure (V2I) communications are displayed.
}
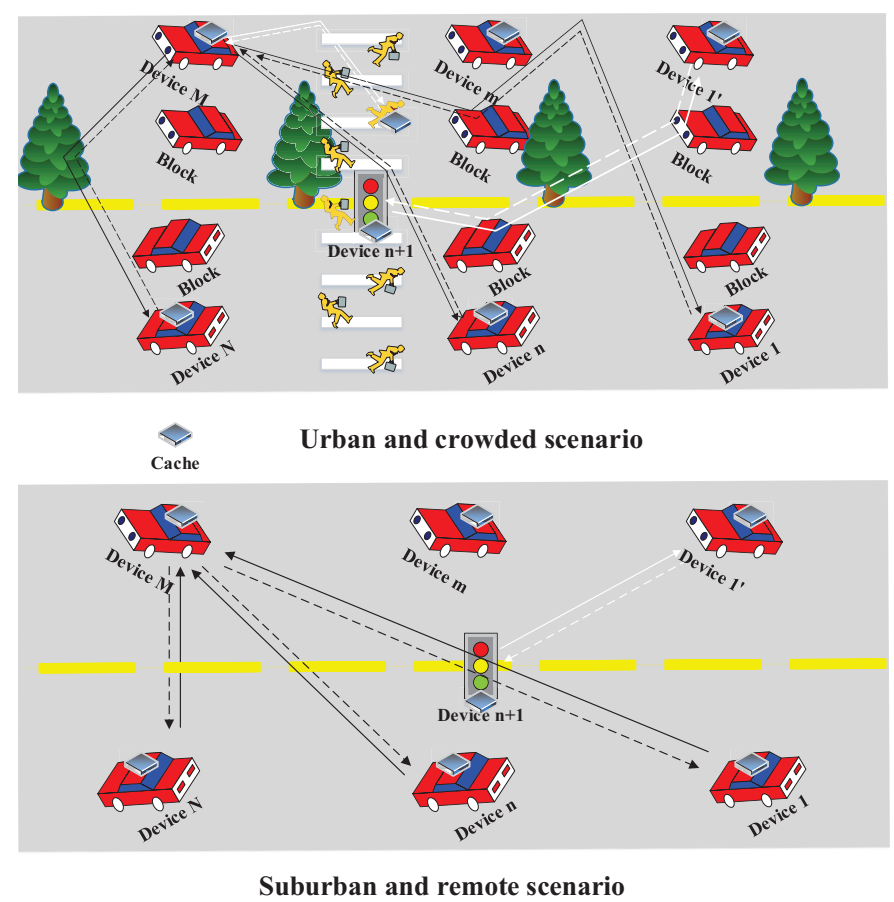

Fig. 1: The $M \leftrightarrow N$ FD-NOMA-based decentralized V2X system model.

interference of $i$-th user is from the $(i+1)$-th user to the $N$ th user [20]. Other co-channel interferences are successfully canceled by the SIC.

\section{B. Ergodic Capacity Analysis in UC Scenario}

We first analyze the system achievable ergodic capacity in UC scenario. Note that we use the superscript $a$ and $c$ to distinguish different scenarios. In UC scenario, Rayleigh channel model is used to describe the transmission environment between source and destination. Probability distribution function (PDF) of the instantaneous signal to interference plus noise ratio (SINR), say, $\gamma_{i, j}$, is given by

$$
f^{a}\left(\gamma_{i, j}\right)=\frac{1}{\bar{\gamma}_{i, j}} e^{-\frac{\gamma_{i, j}}{\bar{\gamma}_{i, j}}},
$$

where $\bar{\gamma}_{i, j}=\frac{\rho \alpha_{i, j}}{\rho\left(\sum_{l=i+1}^{N} \alpha_{i, l}+\eta \alpha_{i, k}\right)+1}$ is the averaged channel power gain of each destination. Here $\rho$ is the SNR. While normalizing the channel noise, we have $\sum_{l=i+1}^{N} \alpha_{i, l}$ the cochannel interference from neighboring users after SIC, $\alpha_{i, k}$ the self-interference from FD uplink, respectively. Additionally, $\eta$ is the coefficient of self-interference with $\eta \in[0,1]$, which makes our expressions versatile to describe different schemes. For instance, in FD-NOMA scheme, large $\eta$ denotes the strong FD self-interference, and small $\eta$ denotes the weak FD selfinterference. On condition that $\eta=0$, the expression reduces to pure NOMA scheme.

As is well known, ergodic capacity is achieved by experiencing all channel fading states. The following equation thus holds

$$
C_{i, j}^{a}=\int_{0}^{+\infty} \log _{2}\left(1+\gamma_{i, j}\right) \frac{1}{\bar{\gamma}_{i, j}} e^{-\frac{\gamma_{i, j}}{\bar{\gamma}_{i, j}}} d \gamma_{i, j}
$$


Theorem 1: In UC scenario, the exact achievable sum ergodic capacity is given as follows

$$
C_{\text {sum }}^{a}=\sum_{i=1}^{M} \sum_{j=1}^{N} e^{\frac{1}{\bar{\gamma}_{i, j}}} E_{1}\left[\frac{1}{\bar{\gamma}_{i, j}}\right] \log _{2} e
$$

where $E_{1}(x)$ is defined as $E_{1}(x)=\int_{x}^{\infty} \frac{e^{-t}}{t} d t$.

Proof: See Appendix A.

Although we have obtained the exact ergodic capacity expression for UC scenario in Theorem 1, this expression is still intractable to be directly used due to the involved exponential integral function. We thereby pursue an approximate closedform expression of the achievable capacity in the sequel. As noticed, the only expression in (3) not given in closed-form is the generalized exponential integral function. In this case, the main focus is to find out an approximate closed-form expression of $E_{1}(x)$, which can be given by the following lemma.

Lemma 1: Closed-form expression (lower bound) of the generalized exponential integral function is

$$
E_{1}(x) \leq 4 \pi \sum_{k=1}^{n+1} \sum_{s=1}^{t+1} a_{k} \sqrt{b_{k}} a_{s} e^{-b_{k} b_{s} x},
$$

where $a_{k}, b_{k}$ are defined as $a_{k}=\frac{\theta_{k}-\theta_{k-1}}{\pi}, b_{k}=\frac{\cot \theta_{k-1}-\cot \theta_{k}}{\theta_{k}-\theta_{k-1}}$. In addition, $\theta_{k}, k \in[0, n+1]$ is given by $0 \leq \theta_{0}<\theta_{1}<$ $\ldots \theta_{k}<\ldots<\theta_{n+1}=\frac{\pi}{2}$. Besides, $a_{s}, b_{s}, \theta_{s}$ are defined with the same method, i.e., $a_{s}=\frac{\theta_{s}-\theta_{s-1}}{\pi}, b_{s}=\frac{\cot \theta_{s-1}-\cot \theta_{s}}{\theta_{s}-\theta_{s-1}}$, and $\theta_{s}, s \in[0, t+1], 0 \leq \theta_{0}<\theta_{1}<\ldots \theta_{s}<\ldots<\theta_{t+1}=\frac{\pi}{2}$. It is also worth noting that the approximation accuracy is controlled by the division of $\frac{\pi}{2}$ with $\theta_{k}$ and $\theta_{s}$ (associate with $\left.a_{s}, b_{s}\right)^{2}$.

Proof: See Appendix B.

The tightness of this approximated closed-form expression is still unsatisfactory. We further notice from Appendix B that in our derivations, the only issue that might bring in difference is the Jensen's inequality. We thus expect a coefficient factor to the closed-form expression to improve the accuracy, i.e., we assume

$$
E_{1}^{\prime}(x)=q 4 \pi \sum_{k=1}^{n+1} \sum_{s=1}^{t+1} a_{k} \sqrt{b_{k}} a_{s} e^{-b_{k} b_{s} x} .
$$

Consequently, our task is to find out a $q$ satisfying

$$
\left|E_{1}^{\prime}(x)-E_{1}(x)\right| \leq \epsilon
$$

In this article, we use $\epsilon=0.00001$. After some manipulations, we notice when $q=\frac{1}{4}$, the above condition is met (e.g., $\left.\left|E_{1}^{\prime}(1)-E_{1}(1)\right|=|0.2193827-0.2193839|=1.2187 \times 10^{-6}\right)$. The approximate closed-form expression of $E_{1}(x)$ then becomes

$$
E_{1}(x) \approx \pi \sum_{k=1}^{n+1} \sum_{s=1}^{t+1} a_{k} \sqrt{b_{k}} a_{s} e^{-b_{k} b_{s} x}
$$

\footnotetext{
${ }^{2}$ In the sequel, equal division of $\frac{\pi}{2}$ is used.
}

Corollary 1: By substituting (7) into (3), we can safely arrive the approximate closed-form expression

$$
C_{\text {sum }}^{a} \approx \pi \log _{2} e \sum_{i=1}^{M} \sum_{j=1}^{N} \sum_{k=1}^{n+1} \sum_{s=1}^{t+1} e^{\left(\frac{1}{\bar{\gamma}_{i, j}}\right)} a_{k} \sqrt{b_{k}} a_{s} e^{\left(-b_{k} b_{s} \frac{1}{\bar{\gamma}_{i, j}}\right)} .
$$

Remark 1: Insights from Corollary 1 is that the system ergodic capacity in UC scenario is determined by $M, N, \bar{\gamma}_{i, j}$, and the capacity increases with $M, N$ increasing. We also notice from this equation that its accuracy is determined by $n, t$. That is, the divisions of $\frac{\pi}{2}$. The validity of this approximate expression will be verified by the following numerical results.

\section{Ergodic Capacity Analysis in SR Scenario}

In this subsection, we focus on the system capacity analysis in the SR scenario. We use $K$ as the Rician factor (which is the ratio between the deterministic and random fast-fading component). It is noticed that we have $K=\frac{r^{2}}{2 \omega^{2}}$, where $r^{2}$ yields the channel gain of $\operatorname{LoS}$ component, $2 \omega^{2}$ is the average channel power gain of all NLoS components. While defining total average power gain $\bar{\gamma}$ and following the prior work in [21], PDF of $\gamma_{i, j}$ can be given as

$$
f^{c}\left(\gamma_{i, j}\right)=\frac{K+1}{\bar{\gamma}_{i, j}} e^{-K-\frac{(K+1) \gamma_{i, j}}{\bar{\gamma}_{i, j}}} I_{0}\left(2 \sqrt{\left.\frac{K(K+1) \gamma_{i, j}}{\bar{\gamma}_{i, j}}\right) .}\right.
$$

Here $I_{0}(\cdot)$ is the modified Bessel function of its first kind and with zeroth order. Following a similar procedure of the previous analysis, we can obtain the exact ergodic capacity expression for SR scenario, which is given by Theorem 2 .

Theorem 2: Exact sum ergodic capacity expression of the FD-NOMA-based decentralized V2X systems in SR scenario is

$$
C_{\text {sum }}^{c}=\sum_{i=1}^{M} \sum_{j=1}^{N} \frac{e^{-K}}{\ln 2} e^{\frac{K+1}{\bar{\gamma}_{i, j}}} \sum_{m=0}^{\infty} \frac{K^{m}}{m !} \sum_{l=1}^{m+1} E_{m-l+2}\left(\frac{K+1}{\bar{\gamma}_{i, j}}\right) .
$$

Here $E_{n}(x)$ is the generalized exponential integral function defined as

$$
E_{n}(x)=\int_{1}^{\infty} \frac{e^{-x t}}{t^{n}} d t(\operatorname{Re}(x)>0),
$$

where $\operatorname{Re}(x)$ yields the real part of $x$ [22].

Proof: See Appendix C.

This expression is intractable to be adopted directly because of the involved infinite factorial and generalized exponential integral expressions. In order to tame the troublesome problems, we give one approximate expression with arbitrary small error by invoking the truncation method in this article.

As shown by Corollary 2, we find that $\sum_{m=0}^{\infty} \frac{K^{m}}{m !} \sum_{q=1}^{m+1} E_{m-q+2}\left(\frac{K+1}{\bar{\gamma}_{i, j}}\right)$ has an upper ceiling approximation. In this case, the system sum ergodic capacity can be given by an approximate expression with much lower computation complexity and arbitrary small error, $\epsilon$. 
Corollary 2: By truncating the infinite series with regard to $T$, the approximate capacity expression becomes

$$
C_{\text {sum }}^{c} \approx \sum_{i=1}^{M} \sum_{j=1}^{N} \frac{e^{-K}}{\ln 2} e^{\frac{K+1}{\bar{\gamma}_{i, j}}} \sum_{m=0}^{T} \frac{K^{m}}{m !} \sum_{q=1}^{m+1} E_{m-q+2}\left(\frac{K+1}{\bar{\gamma}_{i, j}}\right),
$$

where its truncation error can be given as

$$
\sum_{i=1}^{M} \sum_{j=1}^{N} \frac{e^{-K}}{\ln 2} e^{\frac{K+1}{\bar{\gamma}_{i, j}}} \sum_{m=T+1}^{\infty} \frac{K^{m}}{m !} \sum_{q=1}^{m+1} E_{m-q+2}\left(\frac{K+1}{\bar{\gamma}_{i, j}}\right) .
$$

Proof: See Appendix D.

Remark 2: We notice that the accuracy of the approximate expression in (12) is controlled by $T$. In other words, we may obtain an approximate expression with an arbitrary small error when $\sum_{i=1}^{M} \sum_{j=1}^{N} \frac{e^{-K}}{\ln 2} e^{\frac{K+1}{\bar{\gamma}_{i, j}}} \sum_{m=T+1}^{\infty} \frac{K^{m}}{m !} \sum_{q=1}^{m+1} E_{m-q+2}\left(\frac{K+1}{\bar{\gamma}_{i, j}}\right)<$ $\epsilon$. Insight from Corollary 2 has that the system sum ergodic capacity expression is determined by $M, N, \bar{\gamma}_{i, j}$ and $K$. With $M, N$ increasing, the system sum ergodic capacity value always increases. The precise effects of $\bar{\gamma}_{i, j}, K$ to the capacity are still nonintuitive, which will be discussed in the following section.

\section{Numerical Results}

We first perform the Monte Carlo simulations to verify the validity of our analysis in this section. We then perform simulations to exposit the effects of different parameters to the system capacity, and compare the performance between FD-NOMA and NOMA schemes based on the decentralized FD-NOMA-enabled V2X systems. Due to variable parameters, we separately explain the parameters and their values in the following simulations.

We first check the validity of the derived capacity expressions in (8) and (12). For the sake of compactness, we use one source employing the FD-NOMA to serve multiple destinations. We assume the allocated NOMA power variance is growing linearly with a normalized noise variance value (e.g., with 4 users, the NOMA power vector is $\left.\mathbf{a}_{i}=[4,3,2,1]\right)$, where $\left.\mathbf{a}_{i}=\left[\alpha_{i, 1}, \ldots, \alpha_{i, N}\right]\right)$. Additionally, $\eta=0.1, \alpha_{i, k}=5$ are used. As clearly shown by Fig. 2a and Fig. 2b, our analytical results ${ }^{3}$ and the MC results almost exactly coincide, which demonstrates the validity of our analysis. For instance, in Fig. 2a, with $\rho=15 \mathrm{~dB}, 1 \leftrightarrow 4$, the MC and App results are respectively $3.6865,3.6866 \mathrm{Bit} / \mathrm{S} / \mathrm{Hz}$. Under the same condition, as shown in Fig. 2b, MC result and App result are 3.8458, 3.8455 Bit/S/Hz, respectively. The difference is less than $0.001 \mathrm{Bit} / \mathrm{S} / \mathrm{Hz}$ in both scenarios. We also observe that as values of $N, \rho$ increases, the system capacity always increases. By comparing Fig. 2a and Fig. 2b, we notice under the same condition, capacities in SR scenario always outperform the ones in UC scenario (for instance, in $1 \leftrightarrow 3$ case, $\mathrm{SNR}=0 \mathrm{~dB}, C_{\text {sum }}^{c}=125 \% C_{\text {sum }}^{a}$; $\left.\mathrm{SNR}=30 \mathrm{~dB}, C_{\text {sum }}^{c}=103 \% C_{\text {sum }}^{a}\right)$. This is due to the less propagation loss with a dominant LoS path between source and destination in the SR scenario.

\footnotetext{
${ }^{3}$ App: approximate, MC: Monte Carlo.
}

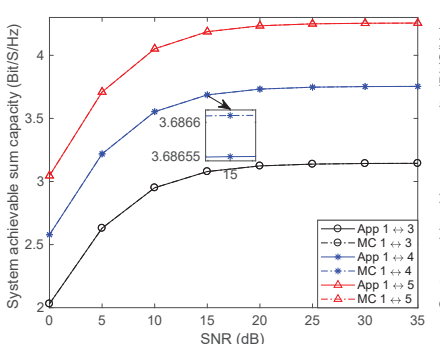

(a) App and $\mathrm{MC}$ results in $\mathrm{UC}$ scenario.

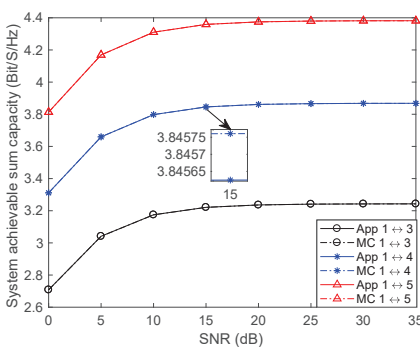

(b) App and MC results in SR scenario.
Fig. 2: Comparison between the App and MC-based results.

In the next step, we try to reveal the effect of Rician factor $K$ to the SR scenario's system sum ergodic capacity. In order to keep $K$ as the only variable, we do some manipulations as follows: 1) we keep all variables consistent except $K$; 2) with normalized noise power value and 3 destinations, we set $\mathbf{a}_{i}=[1,2,3]$. The simulation results of system sum ergodic capacity vs destination number in SR scenario is given in Fig. 3a. As decipted here, with $K$ increasing, system capacity also increases. This is because higher $K$ results in a much stronger LoS component and a much weaker multi-path propagation loss.

Besides the effects of $N, \rho, K$, the effects of $M$ and $\mathbf{a}_{i}$ to the system sum ergodic capacity are verified with the following assumptions: 1) a linearly growing power value with $M=1$ (i.e., $\left.\mathbf{a}_{1}=[0.5,1,1.5], \mathbf{a}_{2}=[1,2,3], \mathbf{a}_{3}=[2,4,6]\right) ; 2$ ) different NOMA power vectors with $M=2$ (i.e., $2 \leftrightarrow 3, \mathbf{a}_{1}, \mathbf{a}_{3}$ denote that two sources are transmitting information to 3 destinations with FD-NOMA, where the NOMA power vector are $\mathbf{a}_{1}, \mathbf{a}_{2}$, respectively). The simulation results are given in Fig. 3b and Fig. 3c. As shown by the solid lines in both figures, increasing the power value leads to better sum ergodic capacity performance, which is due to the increasing SNR. For instance, with $1 \leftrightarrow 3$ and SNR $=20 \mathrm{~dB}$, we have $C_{\text {sum }}^{a}\left(\mathbf{a}_{2}\right)=131 \% C_{\text {sum }}^{a}\left(\mathbf{a}_{1}\right)$. We can also confirm from these two figures that as $M$ increases, the system sum ergodic capacity also increases.

We finally compare the achievable throughputs of the decentralized V2X systems with FD-NOMA, NOMA, FD-OMA and HD-OMA schemes. The results are given in Fig. 4a and Fig. 4b. In these simulations, carrier bandwidth $B=100$ $\mathrm{MHz}, \mathbf{a}_{i}=[3,2,1], \eta=0.1$ and $\alpha_{i, k}=0.1,1,10$ are used. In order to be fair, we average the allocated power in FDOMA and HD-OMA schemes. As is shown here, NOMA schemes display better throughput performance compared to OMA schemes. Moreover, with a smaller value of $\alpha_{i, k}$, FDNOMA outperforms the other schemes (HD-NOMA, FD-OMA, HD-OMA). However, the benefit of FD-NOMA decreases while $\alpha_{i, k}$ increasing, which is because of the increasing FD selfinterference. We also notice even with a higher FD selfinterference value, FD-NOMA outperforms NOMA in low SNR scenario (i.e., $\rho \in[0,5] \mathrm{dB}$ ). This is because in low SNR scenario, channel noise is the dominant factor compared to the 


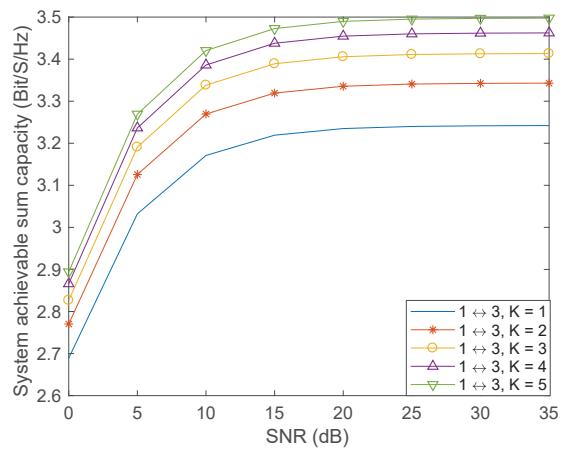

(a) SR scenario capacity performance with different $K$ values.
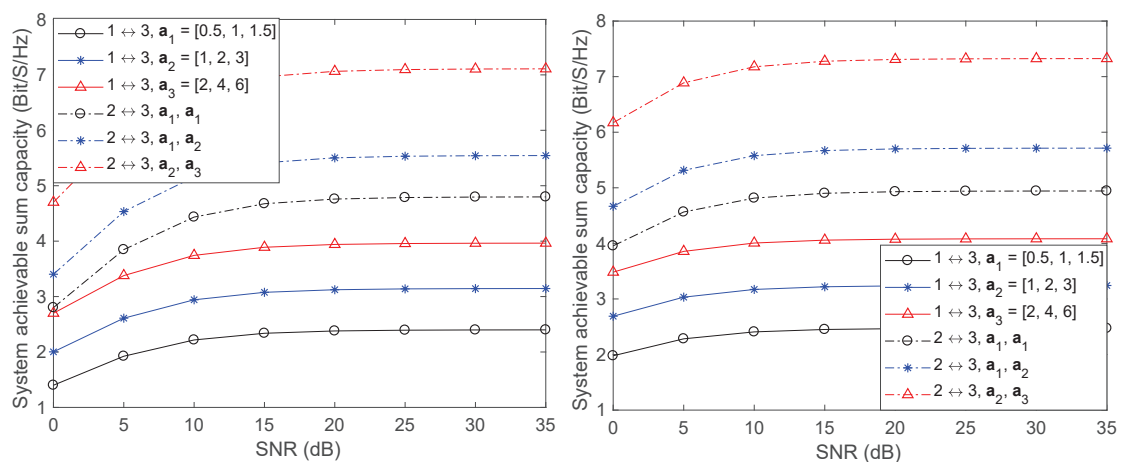

(b) UC scenario capacity performance with (c) UC scenario capacity performance with different power values and source numbers. different power values and source numbers.

Fig. 3: System capacity performance in different scenarios.

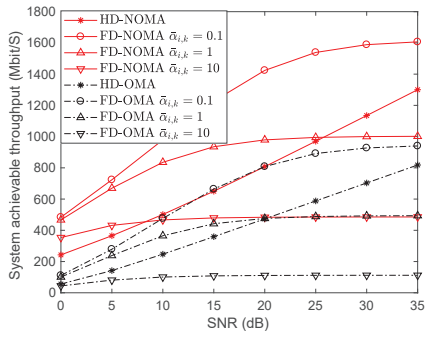

(a) Throughput comparisons in UC scenario.

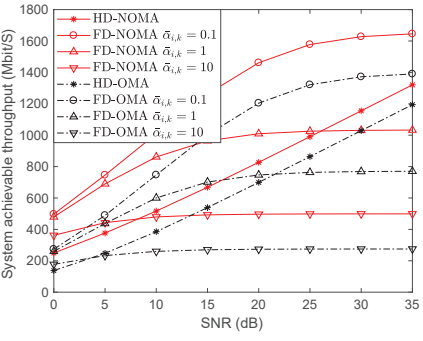

(b) Throughput comparisons in SR scenario.
Fig. 4: System achievable throughput comparisons amongst FDNOMA, NOMA, FD-OMA and HD-OMA schemes in different scenarios.

FD self-interference. In contrast, FD-NOMA self-interference becomes the dominant factor in high SNR scenario, NOMA scheme without FD self-interference thus has a better throughput performance. It is also worth noting that the effective transmission time is limited because of the fast moving V2X devices. FD-NOMA enabled bidirectional transmission thus can greatly reduce the transmission latency compared to other schemes. For example, compared to HD-NOMA and HD-OMA, FD-NOMA only needs a half latency time to transmit the same amount of data by its simultaneous transmission and reception scheme.

\section{CONCLUSION}

In this article, we proposed the FD-NOMA-based decentralized V2X systems. We classified the V2X communications into two typical scenarios, i.e., the UC scenario and the S$\mathrm{R}$ scenario, and then derived the exact system sum ergodic capacity expressions in both scenarios. To tackle down the capacity expression's intractable calculations in both scenarios, we further obtained their simplified approximate expressions. Insights of our analysis are that the accuracy of our simplified approximate expression in UC scenario is determined by the associated division of $\frac{\pi}{2}$ (with respect to exponential integral function $\left(E_{1}(x)\right)$, and the accuracy of simplified approximate expression in SR scenario is determined by the truncation point $T$ (with respect to generalized exponential integral function $\left(E_{n}(x)\right)$. Numerical results demonstrate the validity and effectiveness of our analytical results. Simulation results also demonstrated that the system capacity performance can be enhanced by increasing the number of V2X devices, NOMA power and Rician factor (SR scenario), and the effectiveness of FD-NOMA is determined by the FD self-interference and the channel noise. In addition, FD-NOMA can greatly reduce the system latency compared to other schemes.

\section{APPENDIX A: PROOF OF THEOREM 1}

Firstly, according to the integration by parts method, we have

$$
\begin{aligned}
& \int_{0}^{+\infty} \log _{2}\left(1+\gamma_{i, j}\right) \frac{1}{\bar{\gamma}_{i, j}} e^{-\frac{\gamma_{i, j}}{\bar{\gamma}_{i, j}}} d \gamma_{i, j} \\
& =e^{\frac{1}{\bar{\gamma}_{i, j}} \log _{2} e \frac{1}{\bar{\gamma}_{i, j}} \int_{0}^{+\infty} \frac{e^{-\left(\frac{\gamma_{i, j}}{\bar{\gamma}_{i, j}}+\frac{1}{\bar{\gamma}_{i, j}}\right)}}{\left(\frac{1}{\bar{\gamma}_{i, j}}+\frac{\gamma_{i, j}}{\bar{\gamma}_{i, j}}\right)} d \gamma_{i, j} .}
\end{aligned}
$$

So far the expression is still intractable. In the next step, we recall the alternative generalized exponential integral expression [22] $E_{n}(x)=x^{n-1} \int_{x}^{\infty} \frac{e^{-t}}{t} d t, x>0$, By substituting it into (A.1), and further summarizing the result with $M$ sources and $N$ destinations, we can safely arrive the final expression.

APPENDIX B: PROOF OF LEMMA I

As noticed, $E_{1}(x)$ can be rewritten as

$$
E_{1}(x)=\int_{x}^{\infty} \frac{e^{-t}}{\sqrt{t}} \frac{1}{\sqrt{t}} d t
$$

It is also noticed that the following equality holds [23] $\frac{e^{-t}}{\sqrt{t}}=-2 \sqrt{\pi} \frac{d}{d t}\{Q(\sqrt{2 t})\}$. According to prior work, by adopting the Craig's form, we have $Q(x)=$ $\frac{\theta_{k}-\theta_{k-1}}{\pi} \sum_{k=1}^{n+1} \frac{\int_{\theta_{k-1}}^{\theta_{k}} e^{-\frac{x^{2}}{2 \sin ^{2} \theta}} 1 d \theta}{\int_{\theta_{k-1}}^{\theta_{k}} 1 d \theta}$, where $a_{k}, b_{k}$ are defined as $a_{k}=\frac{\theta_{k}-\theta_{k-1}}{\pi}, b_{k}=\frac{\cot \theta_{k-1}-\cot \theta_{k}}{\theta_{k}-\theta_{k-1}}$. Then by substituting the Jensen's inequality [24], we have

$$
Q(x) \geq \frac{\theta_{k}-\theta_{k-1}}{\pi} \sum_{k=1}^{n+1} a_{k} e^{-\left(\frac{x^{2}}{2\left(\theta_{k}-\theta_{k-1}\right)} \int_{\theta_{k-1}}^{\theta_{k}} \frac{1}{\sin ^{2} \theta} d \theta\right)} .
$$


Additionally, it is worth noting that $\theta_{k}, k \in[0, n+1]$ is given by $0 \leq \theta_{0}<\theta_{1}<\ldots \theta_{k}<\ldots<\theta_{n+1}=\frac{\pi}{2}$ [23], [24]. The approximate accuracy of this lower bound expression is controlled by the interval gap between each pair of $\left[\theta_{k-1}, \theta_{k}\right]$. Moreover, it is known that $\int \sin ^{-2} x d x=-\cot x+C$. Substituting it into (B.2), we thus have $Q(x) \approx \sum_{k=1}^{n+1} a_{k} e^{-\frac{x^{2} b_{k}}{2}}$. Finally, $E_{1}(x)$ can be given as

$$
E_{1}(x) \approx 4 \pi \sum_{k=1}^{n+1} \sum_{s=1}^{t+1} a_{k} \sqrt{b_{k}} a_{s} e^{-b_{k} b_{s} x} .
$$

\section{Appendix C: Proof of Theorem 2}

As is known, the modified Bessel function of the zeroth order expression is $I_{0}(x)=\sum_{m=0}^{\infty} \frac{\left(\frac{x}{2}\right)^{2 m}}{m ! \Gamma(m+1)}$. We also notice

that $\int_{0}^{\infty} \ln (1+\kappa x) \frac{x^{z-1}}{e^{\beta x}} d x=\frac{\Gamma(z) e^{\frac{\beta}{\kappa}}}{\beta^{z}} \sum_{l=1}^{z} E_{z-l+1}\left(\frac{\beta}{\kappa}\right)$. By adopting them with regard to ergodic capacity definition, we thus have

$$
C_{i, j}^{c}=\frac{e^{-K}}{\ln 2} e^{\frac{K+1}{\bar{\gamma}_{i, j}}} \sum_{m=0}^{\infty} \frac{K^{m}}{m !} \sum_{q=1}^{m+1} E_{m-q+2}\left(\frac{K+1}{\bar{\gamma}_{i, j}}\right) .
$$

\section{ApPendix D: Proof of COROLlary 2}

The remaining section after a truncation with regard to $T$ is

$$
\sum_{i=1}^{M} \sum_{j=1}^{M} \frac{e^{-K}}{\ln 2} e^{\frac{K+1}{\bar{\gamma}_{i, j}}} \sum_{m=T+1}^{\infty} \frac{K^{m}}{m !} \sum_{q=1}^{m+1} E_{m-q+2}\left(\frac{K+1}{\bar{\gamma}_{i, j}}\right) .
$$

As shown here, approximate error mainly comes from the infinite expression series with regard to $m$. According to prior work in [25], [26], $E_{n}(x)$ monotonically decreasing in $n$ giving equal $x$. In this case, by putting $\sum_{i=1}^{M} \sum_{j=1}^{M} \frac{e^{-K}}{\ln 2} e^{\frac{K+1}{\bar{\gamma}_{i, j}}}$ part aside, we have $\sum_{m=T+1}^{\infty} \frac{K^{m}}{m !} \sum_{q=1}^{m+1} E_{m-q+2}\left(\frac{K+1}{\bar{\gamma}_{i, j}}\right)<$ $\sum_{m=T+1}^{\infty} \frac{K^{m}}{m !} \frac{(m+1)(m+2)}{2} E_{1}\left(\frac{K+1}{\bar{\gamma}_{i, j}}\right)$. It is noticed that giving constant values of $\gamma_{i, j}$ and $K, E_{1}\left(\frac{K+1}{\bar{\gamma}_{i, j}}\right)$ then becomes a constant coefficient. Consequently, we focus on the function $f(x)=\frac{K^{x}}{x !} \frac{(x+1)(x+2)}{2}$. By some mathematical manipulations, it is found that there existing $x^{\prime}$, so that $f^{\prime}\left(x^{\prime}\right)=0$ with $f^{\prime \prime}\left(x^{\prime}-\right)>0, f^{\prime \prime}\left(x^{\prime}+\right)<0$. Additionally, observation has that $f(x)$ rapidly converges to 0 after $x^{\prime}$ (e.g., $f(100)=$ $\left.6.9966 \times 10^{-125}\right)$. This gives approximate capacity expression of (12) with an arbitrary small error $\epsilon$.

\section{REFERENCES}

[1] J. B. Kenney, "Dedicated short-range communications (DSRC) standards in the united states," Proc. IEEE, vol. 99, no. 7, pp. 1162-1182, Jul. 2011.

[2] B. Walke, W. Mende, and G. Hatziliadis, "CELLPAC: A packet radio protocol applied to the cellular GSM mobile radio network," in IEEE VTC, May 1991, pp. 408-413.

[3] Z. Xu, X. Li, X. Zhao, M. H. Zhang, and Z. Wang, "DSRC versus 4GLTE for connected vehicle applications: A study on field experiments of vehicular communication performance," J. Adv. Transp., vol. 2017, pp. $1-10$, Aug. 2017

[4] G. Americas, "Cellular V2X communications towards 5G," 5G Americas, Tech. Rep., Mar. 2018

[5] H. Ji, S. Park, J. Yeo, Y. Kim, J. Lee, and B. Shim, "Ultra-reliable and lowlatency communications in 5G downlink: Physical layer aspects," IEEE Wireless Commun., vol. 25, no. 3, pp. 124-130, Jun. 2018.
[6] Y. Fan, L. Yang, D.-L. Zhang, G. Han, and D. Zhang, "An angle rotateQAM aided differential spatial modulation for 5G ubiquitous mobile networks (accept)," Mobile Networks and Applications, June 2019.

[7] X. Yue, Y. Liu, S. Kang, A. Nallanathan, and Z. Ding, "Exploiting full/half-duplex user relaying in NOMA systems," IEEE Trans. Commun., vol. 66, no. 2, pp. 560-575, Feb. 2018.

[8] A. Sabharwal, P. Schniter, D. Guo, D. W. Bliss, S. Rangarajan, and R. Wichman, "In-band full-duplex wireless: Challenges and opportunities," IEEE J. Sel. Areas Commun., vol. 32, no. 9, pp. 1637-1652, Sep. 2014.

[9] Z. Ding, P. Fan, and H. V. Poor, "On the coexistence between full-duplex and NOMA [early access:] https://ieeexplore.ieee.org/document/8306094/," IEEE Wireless Commun. Lett., Mar. 2018.

[10] M. F. Kader, S. Y. Shin, and V. C. M. Leung, "Full-duplex non-orthogonal multiple access in cooperative relay sharing for $5 \mathrm{G}$ systems," IEEE Trans. Veh. Technol., vol. 67, no. 7, pp. 5831-5840, Jul. 2018.

[11] B. Di, L. Song, Y. Li, and G. Y. Li, "Non-orthogonal multiple access for high-reliable and low-latency V2X communications in 5G systems," IEEE J. Sel. Areas Commun., vol. 35, no. 10, pp. 2383-2397, Oct. 2017.

[12] B. W. Khoueiry and M. R. Soleymani, "An efficient NOMA V2X communication scheme in the internet of vehicles," in IEEE VTC, Jun. 2017, pp. 1-7.

[13] M. Yang, S. W. Jeon, and D. K. Kim, "Interference management for inband full-duplex vehicular access networks," IEEE Trans. Veh. Technol., vol. 67, no. 2, pp. 1820-1824, Feb. 2018

[14] M. D. Yacoub, "The $\kappa-\mu$ distribution and the $\eta-\mu$ distribution," IEEE Antennas and Propag. Mag., vol. 49, no. 1, pp. 68-81, Feb. 2007.

[15] N. Bhargav, S. L. Cotton, and D. E. Simmons, "Secrecy capacity analysis over $\kappa \mathrm{c} \mu$ fading channels: Theory and applications," IEEE Trans. Commun., vol. 64, no. 7, pp. 3011-3024, Jul. 2016.

[16] X. Li, J. Li, L. Li, J. Jin, J. Zhang, and D. Zhang, "Effective rate of miso systems over $\kappa-\mu$ shadowed fading channels," IEEE Access, vol. 5, pp 10605-10611, Jun. 2017.

[17] D. Zhang, Z. Zhou, S. Mumtaz, J. Rodriguez, and T. Sato, "One integrated energy efficiency proposal for 5G IoT communications," IEEE Internet Things J., vol. 3, no. 6, pp. 1346-1354, Dec. 2016.

[18] R. J. C. Bultitude and G. K. Bedal, "Propagation characteristics on microcellular urban mobile radio channels at $910 \mathrm{MHz}$," IEEE J. Sel. Areas Commun., vol. 7, no. 1, pp. 31-39, Jan. 1989.

[19] Q. Zhang, T. Q. S. Quek, and S. Jin, "Scaling analysis for massive MIMO systems with hardware impairments in Rician fading [early access:] https://ieeexplore.ieee.org/document/8350341/," IEEE Trans. Wireless Commun., Apr. 2018

[20] Y. Liu, Z. Ding, M. Elkashlan, and H. V. Poor, "Cooperative nonorthogonal multiple access with simultaneous wireless information and power transfer," IEEE J. Sel. Areas Commun., vol. 34, no. 4, pp. 938953, Apr. 2016

[21] H. C. Yang and M. S. Alouini, Order Statistics in Wireless Communications: Diversity, Adaptation, and Scheduling in MIMO and OFDM Systems. New York, USA: Cambridge University Press, 2011.

[22] I. S. Gradshteyn and I. M. Ryzhik, Table of Integrals, Series and Products, 8th ed. London, UK: Academic Press, 2015.

[23] A. A. Alkheir and M. Ibnkahla, "An accurate approximation of the exponential integral function using a sum of exponentials," IEEE Commun. Lett., vol. 17, no. 7, pp. 1364-1367, Jul. 2013.

[24] M. Wu, X. Lin, and P. Y. Kam, "New exponential lower bounds on the Gaussian Q-Function via Jensen's inequality," in IEEE VTC Spring, May 2011, pp. 1-5.

[25] J. Zhang, L. Dai, X. Zhang, E. Bjönson, and Z. Wang, "Achievable rate of Rician large-scale MIMO channels with transceiver hardware impairments," IEEE Trans. Veh. Technol., vol. 65, no. 10, pp. 8800-8806, Oct. 2016.

[26] C. Chiccoli, S. Lorenzutta, and G. Maino, "Recent results for generalized exponential integrals," Comput. Math. Appl., vol. 19, no. 5, pp. 21 - 29, 1990. 